\title{
Integrability of motion around galactic razor-thin disks
}

\author{
Ronaldo S. S. Vieira • Javier Ramos-Caro
}

Received: date / Accepted: date

\begin{abstract}
We consider the three-dimensional bounded motion of a test particle around razor-thin disk configurations, by focusing on the adiabatic invariance of the vertical action associated with disk-crossing orbits. We find that it leads to an approximate third integral of motion predicting envelopes of the form $Z(R) \propto$ $[\Sigma(R)]^{-1 / 3}$, where $R$ is the radial galactocentric coordinate, $Z$ is the z-amplitude (vertical amplitude) of the orbit and $\Sigma$ represents the surface mass density of the thin disk. This third integral, which was previously formulated for the case of flattened 3D configurations, is tested for a variety of trajectories in different thin-disk models.
\end{abstract}

Keywords Dynamics of galaxies - Razor-thin disks · Disk-crossing orbits · Vertical stability · Third integral · Adiabatic approximation

\section{Introduction}

Many galaxies in the Universe are nearly axisymmetric, with a mass distribution formed by several components: a thin disk, a central bulge and a surrounding halo. In consequence, there is a number of mass models incorporating one or all of these features, depending on the particular situation (Freeman 1970; Kent 1986, 1987; Sofue et al. 2009). But, in all cases, the disk component represents a significant percentage of the galactic mass, taking into account that the main part of the stellar population is located there. For systems in which this component is highly flattened, we can use thin disks in the limit of negligible thickness (razor-thin disks) in order to provide preliminary, but simple and tractable, models for the stellar

Ronaldo S. S. Vieira

Instituto de Astronomia, Geofísica e Ciências Atmosféricas, Universidade de São Paulo, 05508-090, São Paulo, SP, Brazil

E-mail: rss.vieira@usp.br

Javier Ramos-Caro

Departamento de Física, Universidade Federal de São Carlos, 13565-905, SP, Brazil

E-mail: javier@ufscar.br 
mass distribution. In consequence, the formulation and characterization of thindisk models have been, for decades, an issue of interest in galactic dynamics (see for example Hunter 1963; Morgan and Morgan 1969; Hunter 2003, 2005; González and Reina 2006; Pedraza et al. 2008, as well as Binney and Tremaine 2008 and references therein).

Razor-thin disks (RTDs) have also been used in other branches of astrophysics. These models can be used to describe self-gravitating disks around a central black hole (see Lemos and Letelier 1994; Semerák and Suková 2010; Lora-Clavijo et al. 2010) and self-gravitating rings (Letelier 2007; Vogt and Letelier 2009; Iorio 2012). The last issue was partially encompassed in Ramos-Caro et al. (2011), for instance, where a linear stability study of the monopole-ring system was performed by using superpositions of Morgan \& Morgan disks. Similar studies were conducted by the authors in the Newtonian realm of galactic dynamics (Ramos-Caro et al. 2008; Pedraza et al. 2008).

The analysis of the motion of test particles in the gravitational field generated by these axially symmetric configurations is a fundamental issue, which helps us to characterize the dynamics in a given model and establish predictions in situations where they can be applied. For example, the study of orbits in the equatorial plane can improve our understanding about the dynamics of intragalactic stellar motion or the flow of particles in accretion disks around black holes. On the other hand, the integrals of motion associated with three-dimensional orbits are a fundamental key to find the galaxy's distribution function in stationary state (Binney and Tremaine 2008). They set the basis for the formulation of dynamical models for the Galaxy (de Zeeuw 1987; Binney 2010; Binney and McMillan 2011; Binney 2012; Binney and Sanders 2014).

In this paper we study three-dimensional bounded motion of test particles around razor-thin distributions of matter, by focusing on the problem of the socalled disk-crossing orbits, i.e. trajectories which cross back and forth through the stellar disk. Numerical experiments reveal prominent regions of KAM curves in the corresponding phase space, suggesting the existence of a third (non-classical) integral of motion (Saa and Venegeroles 1999; Hunter 2003, 2005; Ramos-Caro et al. 2008). This is an interesting fact, taking into account that smooth versions of KAM theorem cannot be applied to such distributions, due to the discontinuity introduced by the razor-thin layer. The principal motivation of this work is to provide a tractable expression for the non-analytical integral of motion governing disk-crossing particles. The results we will show here, suitable for situations with discontinuous gravitational fields, can be considered as a complement to the analysis conducted in Vieira and Ramos-Caro (2014), applicable only to continuous fields due to smooth matter distributions.

The problem of finding an analytical expression for the third integral of motion governing the orbits of stars in a galaxy has been calling the attention of the astrophysics community for decades (Contopoulos 1960, 1963, 2001, 2002; Hietarinta 1987; Bienaymé and Traven 2013; Bienaymé et al. 2015). This issue can be addressed in a simple way for some elementary situations, as for example, the description of quasi-circular orbits around the equatorial plane of a three-dimensional flattened distribution. In such a case, the epicyclic approximation holds and, by invoking the adiabatic invariance of the corresponding vertical action, one can construct an approximate formula for the third integral, $I_{3}$, in cylindrical coordinates 
$(R, \varphi, z)$, evaluated at the orbit's envelope $Z(R)$ (Binney and Tremaine 2008):

$$
I_{3}=Z(R) \Phi_{z z}^{1 / 4}(R, 0),
$$

where $Z(R)$ is the orbit's vertical amplitude at a radial coordinate $R$, and $\Phi_{z z}$ represents the second derivative of the gravitational potential with respect to $z$. Unfortunately, the range of validity of such a formula is very limited and, more important, it is not applicable to potentials generated by RTDs, for which $\Phi_{z z}$ cannot be defined inside the matter distribution.

We present in this paper a new approximate third integral of motion, valid for disk-crossing orbits in a potential due to a RTD (a different approximate third integral for a class of $C^{0}$ systems was presented in Varvoglis 1985). This integral describes the shape of nearly equatorial orbits in terms of $\Sigma$, the surface matter density of the razor-thin layer. The approach begins with a presentation of the stability analysis of equatorial circular orbits belonging to the razor-thin distribution of matter, in order to establish stability criteria appropriated to situations involving field discontinuities. The results of this analysis are incorporated in the description of adiabatic invariants, obtaining an expression as simple as (1) when evaluated at the orbit's envelope:

$$
I_{3}=Z(R) \Sigma^{1 / 3}(R),
$$

which means that the vertical amplitude $Z$, at a galactocentric radius $R$, is determined by the surface mass distribution $\Sigma$ through a relation of the form $Z \propto \Sigma^{-1 / 3}$, setting the shape of the envelope traced in the configuration space $(R, z)$ (also called meridional plane). Remarkably, the envelopes of nearly equatorial diskcrossing orbits are determined exclusively by the surface density of the razor-thin disk, even when there are other matter sources around it (halo, thick disk, etc).

In the following, we present the procedure to obtain formula (2), which represents the RTD-version of formula (1). At first, we present a brief revision on the formulation of models including RTDs (sec. 2), in order to set the properties of the gravitational potential determining the test-particle motion, which will be addressed in sec. 3 . There, the main problem will be to study the stability of equatorial circular orbits under perturbations in $R$ - (radial) and $z$ - (vertical) directions, in order to obtain stability criteria suitable to regions belonging to the thin layer. Then, we incorporate such criteria in the description of disk-crossing orbits. In particular, we show that a variety of these orbits can be described by an approximate third integral of motion given by Eq. (2) (sec. 4). We test our results with numerical experiments in different razor-thin disk models (sec. 5). Finally, we present our conclusions in sec. 6 .

\section{Galactic Models via RTDs}

As it was pointed out in the Introduction, many galaxies are modeled as an axisymmetric RTD surrounded by an axisymmetric 3D smooth density distribution, which is symmetric with respect to the equatorial plane. The total density distribution can be written as

$$
\rho(R, z)=\Sigma(R) \delta(z)+\rho_{s}(R, z)
$$


where $\delta$ is the Dirac delta, $\Sigma$ is the surface density distribution of the RTD and $\rho_{s}$ describes the surrounding matter. The gravitational potential of the system is

$$
\Phi(R, z)=\Phi_{\Sigma}(R, z)+\Phi_{s}(R, z),
$$

where $\Phi_{\Sigma}$ and $\Phi_{s}$ are the contributions due to $\Sigma$ and $\rho_{s}$, respectively, in such a way that they obey the field equations $\nabla^{2} \Phi_{\Sigma}=4 \pi G \Sigma(R) \delta(z)$ and $\nabla^{2} \Phi_{s}=4 \pi G \rho_{s}$.

The assumption that the distribution $\rho_{s}$ is symmetric with respect to the equatorial plane implies the same reflection symmetry for the gravitational potential, i.e. $\Phi(R, z)=\Phi(R,-z)$. Then, the $z$-dependence of $\Phi$ is solely on $|z|$ and, from Poisson's equation, $\Sigma$ is given by

$$
\Sigma(R)=\left.\frac{1}{2 \pi G} \frac{\partial \Phi}{\partial|z|}\right|_{z=0},
$$

which is equivalent to the expression appearing in González and Reina (2006) and references therein.

\section{Stability of equatorial circular orbits in RTD models}

The motion of test particles under the action of $\Phi(R, z)$ can be described by the Hamiltonian $H=\left(P_{R}^{2}+P_{z}^{2}\right) / 2+\Phi_{\text {eff }}$ (per unit mass), where $P_{R}=\dot{R}, P_{z}=\dot{z}$ and $\Phi_{\text {eff }}=\Phi(R, z)+\ell^{2} /\left(2 R^{2}\right)$ is the effective potential, determined by $\ell$, the $z$-component of the particle's specific angular momentum. Consider an equatorial circular orbit of radius $R$ under the action of a small vertical perturbation ${ }^{1}$. This perturbation can be seen as an instantaneous vertical increase $v_{0 z}$ in the velocity of the particle, which does not affect the value of $\ell$. In order to study the evolution of the perturbation in the course of time, we have to use the $z$-equation of motion, $\ddot{z}=-\partial \Phi / \partial z$, which can be written as

$$
\ddot{z}=-[2 \Theta(z)-1] \frac{\partial \Phi}{\partial|z|},
$$

where $\Theta$ is the Heaviside function. From the above expression it can be noticed that the vertical perturbation will remain small if and only if $[\partial \Phi / \partial|z|]_{z=0}>0$. It guarantees that, if $v_{0 z}$ is small enough, the perturbed trajectory will oscillate around the original one for long times. This assertion can be seen, for instance, from the expansion of $\Phi_{e f f}(R, z)$ in powers of $|z|$ :

$$
\Phi_{e f f}(R, z)=\Phi_{e f f}(R, 0)+\frac{\partial \Phi}{\partial|z|}(R, 0)|z|+\mathcal{O}\left(|z|^{2}\right) .
$$

The particle will always cross the disk with a velocity whose vertical component has absolute value $\left|v_{0}\right|$. This is a consequence of: (i) the conservation of mechanical energy; (ii) the assumption that the $R$-coordinate does not change in the process and (iii) the fact that the discontinuity is only in acceleration. So, just after crossing the disk, the particle will also have a velocity with vertical component of magnitude $\left|v_{0 z}\right|$, which means that motion after crossing the disk will have the same behavior

\footnotetext{
1 Here, the term "small" means small enough to neglect variations in the projection of the orbit on the $z=0$ plane.
} 
as motion before crossing it. Therefore, the perturbed orbit remains oscillatory around the original one for sufficiently small initial vertical velocity $v_{0 z}$.

According to (5), condition $[\partial \Phi / \partial|z|]_{z=0}>0$ can be written in terms of the surface mass density $\Sigma$. Then we can state that in a Newtonian razor-thin disk model, a necessary and sufficient condition for vertical stability of a circular orbit of radius $R$ is $\Sigma(R)>0$. But we know that all the disk models, intended to represent realistic matter distributions, satisfy such a condition. Then we can state that the circular equatorial orbits in RTD models are always stable under small vertical perturbations.

By introducing (7) in the equations of motion, it is possible to estimate, for a sufficiently small vertical perturbation, the characteristic period $T$ and amplitude $Z$ of the corresponding oscillations around the equatorial plane. For the period we obtain $T=2 v_{0 z} /(\pi G \Sigma(R))$, whereas for the amplitude of the oscillation, we can write $Z=v_{0 z}^{2} /(4 \pi G \Sigma(R))$. As expected, the amplitude of oscillations is inversely proportional to the surface mass density (but not to the volumetric mass density of the surrounding matter, if present).

As it is well known, radial stability is guaranteed once Rayleigh's criterion is satisfied (Landau and Lifshitz 2007; Letelier 2003).

\section{Approximate integrability of motion near a stable circular orbit}

We remarked in the Introduction that motion is integrable near a stable circular orbit, a well-known result in the case of smooth density distributions. There are many evidences of this general behavior for razor-thin disks in the literature (Saa and Venegeroles 1999; Hunter 2003, 2005; Pedraza et al. 2008; Ramos-Caro et al. 2008; González et al. 2010; Ramos-Caro et al. 2011), where it is found numerically, by means of Poincaré sections, that motion is integrable around what appears to be a stable point of the effective potential - corresponding to a stable circular orbit in the equatorial plane. These evidences also show that the integrable domain goes well beyond the neighborhood of the stable circular orbit, reaching regions where the approximation of a separable potential is not valid anymore.

The integrability of motion near the stable circular orbit for density profiles of the form (3) follows from the separability of the effective potential near the stable point $\left(R_{o}, 0\right)$. In fact, up to first order in $|z|$, we have

$$
\begin{aligned}
\Phi_{\text {eff }}(R, z) & \approx \Phi_{\text {eff }}(R, 0)+\frac{\partial \Phi_{\text {eff }}}{\partial|z|}(R, 0)|z| \\
& =\Phi_{\text {eff }}(R, 0)+2 \pi G \Sigma(R)|z| .
\end{aligned}
$$

Since the orbit is radially stable, we can approximate $\Sigma(R) \approx \Sigma\left(R_{o}\right)$, discarding higher-order terms. Thus,

$$
\Phi_{\text {eff }}(R, z) \approx \Phi_{\text {eff }}(R, 0)+2 \pi G \Sigma\left(R_{o}\right)|z|
$$

and the corresponding approximate Hamiltonian, $H=\left(P_{R}^{2}+P_{z}^{2}\right) / 2+\Phi_{\text {eff }}$, is separable. In this way, we obtain by quadratures two independent approximate integrals of motion, namely the approximate action variables, near $\left(R_{o}, 0\right)$ : one for the $R$-coordinate, $J_{R}$, and another for the $z$-coordinate, $J_{z}$, which is of special interest here. 
It can be shown that the vertical action $J_{z}$ can be written as

$$
J_{z}=\frac{4 \sqrt{2}}{3 \pi}\left[2 \pi G \Sigma\left(R_{o}\right)\right]^{1 / 2} Z^{3 / 2}
$$

This expression helps us to study the effects of adiabatic variations in the approximate potential of Eq. (9). Following the procedure presented in section 3.6 of Binney and Tremaine (2008), we obtain an expression that relates the $z$-amplitudes of a given orbit at different values of the radial coordinate $(R \neq \tilde{R})$ :

$$
\frac{Z(R)}{Z(\tilde{R})}=\left[\frac{\Sigma(\tilde{R})}{\Sigma(R)}\right]^{1 / 3}
$$

This relation, which is the same as (2), determines the "envelope" of the orbit in the meridional plane. It works well whenever the vertical oscillations are faster than radial oscillations, as in the case of orbits which deviate only slightly from the equatorial plane. Equation (11), valid for razor-thin disks (and previously presented in Vieira and Ramos-Caro 2015), is the analogue of the corresponding well-known relation for smooth potentials (eq. (1), which is the same as eq. 3.279 of Binney and Tremaine 2008).

Finally, we point out that the approximated third integral, associated with $J_{z}$, takes the form

$$
I_{3}=\frac{1}{2 \pi G}[\Sigma(R)]^{-2 / 3}\left[\frac{1}{2} P_{z}^{2}+2 \pi G \Sigma(R)|z|\right]
$$

in terms of the phase-space coordinates, which can be compared with other approximated expressions. It can be shown that the above relation implies (11) when evaluated along the orbit's envelope $P_{z}=0$, relating the vertical amplitude to the radial coordinate. The vertical action $J_{z}$ relates to $I_{3}$ by

$$
J_{z}=\frac{8 G^{1 / 2}}{3 \pi^{1 / 2}}\left[I_{3}\right]^{3 / 2}
$$

(see Eq. (10)), so its expression in terms of phase-space variables is obtained using Eq. (12).

\section{Examples via numerical experiments}

In order to check the validity of (11), we can compare the envelopes that it describes with the envelopes of numerically calculated off-equatorial orbits in a given potential $\Phi_{\text {eff }}$. Here we perform numerical experiments for the motion of test particles around mass distributions of the form (3), by considering several razor-thin disk potentials. These "nearly circular" orbits may be obtained by considering orbits with relative energy much smaller than the binding energy of the particle: $\Delta E \equiv\left|E-\Phi_{\text {eff }}\left(R_{o}, 0\right)\right| /\left|\Phi_{\text {eff }}\left(R_{o}, 0\right)\right| \ll 1$. It is expected that, in this situation, the predicted $Z(R)$ matches the real envelope, since for this energy range the orbits would be bounded to a region in the meridional plane where the approximations made in the former section are reasonable. On the other hand, to check the validity of (12), we compute the time series of $I_{3}$ along the interval of integration of the 

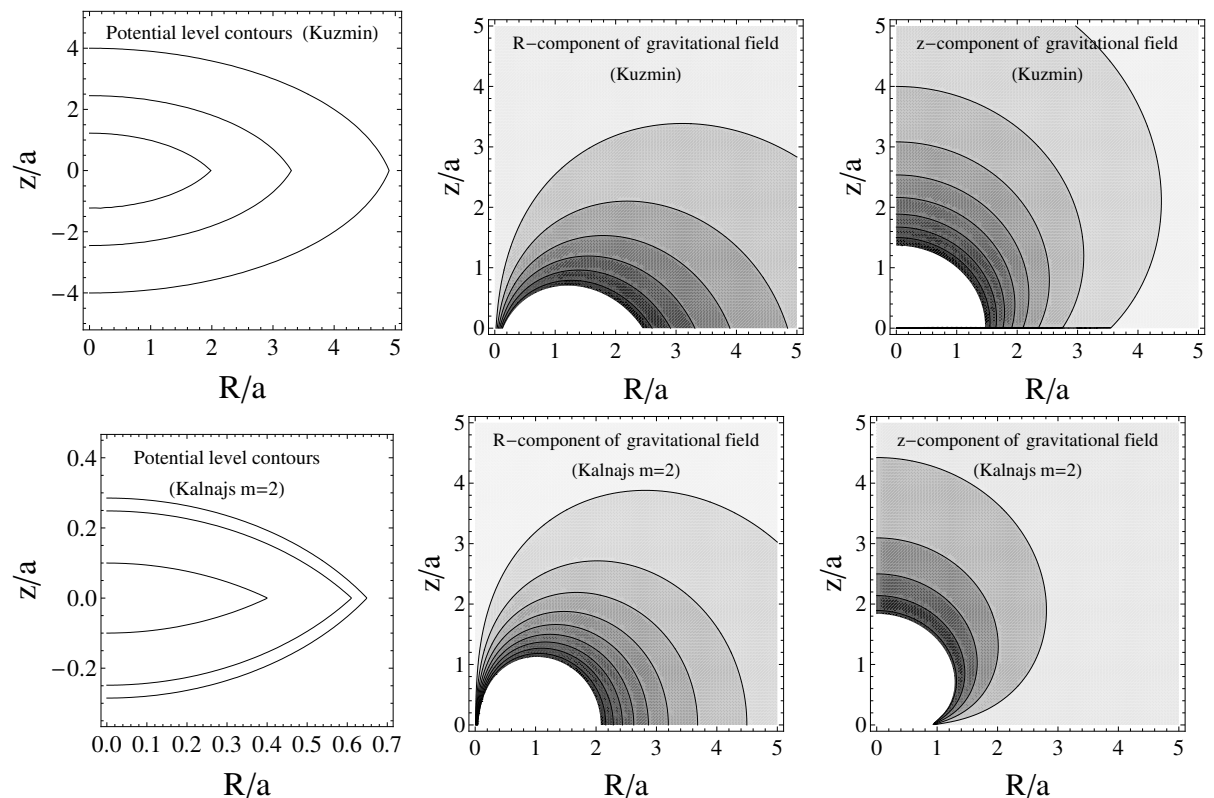

Fig. 1 We show the potential and the modulus of $R$ and $z$ components of gravitational field (force per unit mass) corresponding to the two disks considered here: Kuzmin (top panels) and Kalnajs $m=2$ (bottom panels). Darker regions show higher values of the components, except for white central regions representing its maximum values.

orbits and compare the surfaces of section of numerically integrated orbits with the phase-space curves associated with $I_{3}$.

We also analyzed a number of situations in which the integrated orbits are far from being considered as "nearly equatorial" or "nearly circular", so we could estimate the range of validity of (11). Apart from the fact that, as we increase the energy of the system, the vertical amplitude of the corresponding orbit tends to grow (as well as its radial range), going beyond the expected region of validity of (11), there is also another phenomenon which would invalidate the predictions of the approximate third integral: as energy grows, the nonlinearity of the Hamiltonian flow implies that the secondary resonance islands in phase space also tend to increase in size. Since the adiabatic approximation considers a separable potential, it is likely that this approximation would break down near these islands (whose orbits in the meridional plane would deviate only slightly from the corresponding periodic orbits). In fact, this is what we found in all the cases analyzed, as we describe next.

We present the results of numerically calculated orbits in three cases: (i) the Kuzmin disk, (ii) the second member of the family of generalized Kalnajs disks (in figure 1 we illustrate the potential and gravitational field for these models) and (iii) the Kuzmin disk surrounded by a Plummer halo. By introducing the halo, one might see how the surrounding structure around the disk interferes in the prediction of (11), which depends solely on the disk's surface density. 

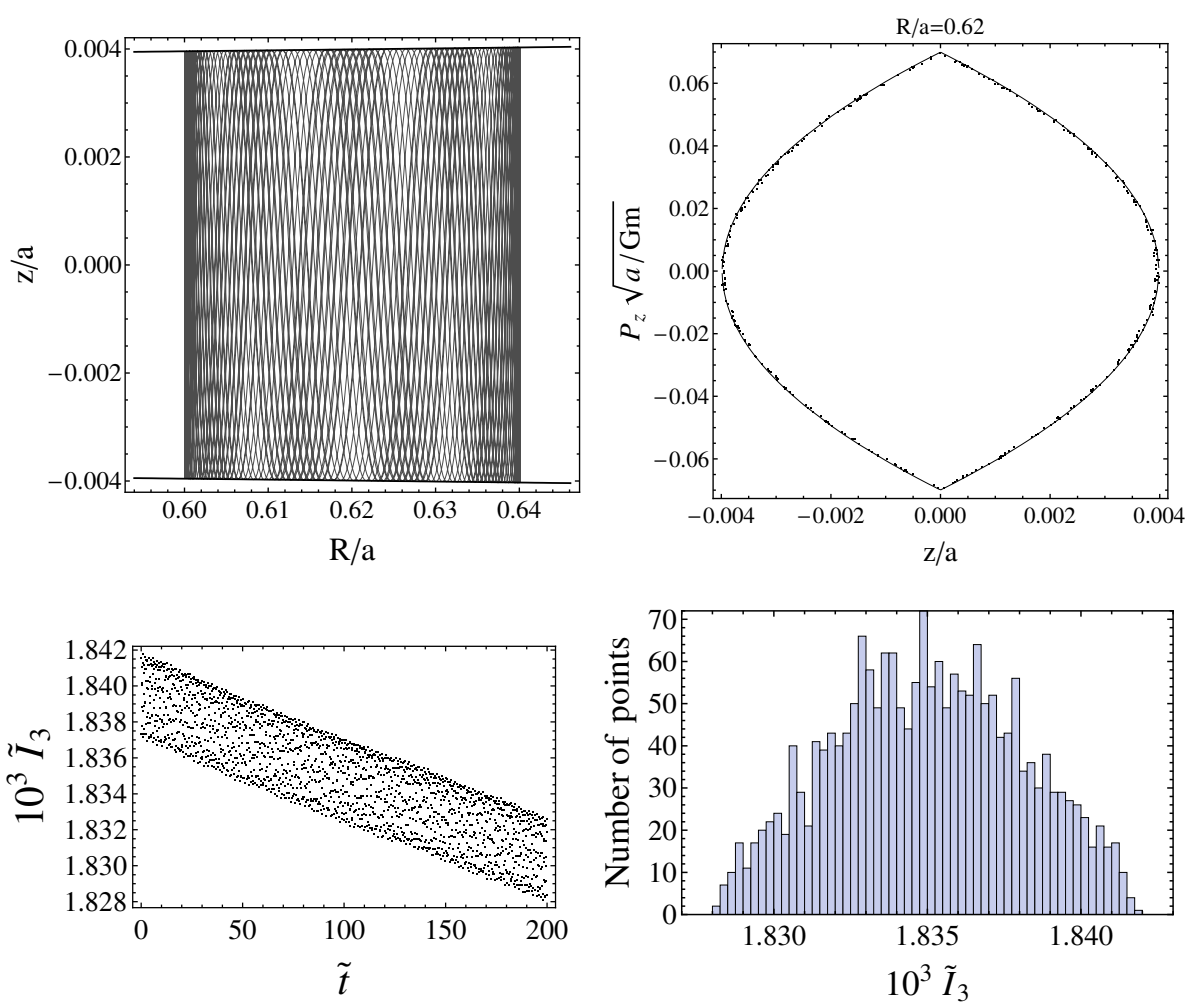

Fig. 2 Top, left: Orbit in Kuzmin's potential shown in the meridional plane. It has initial conditions $R_{o} / a=0.6, P_{R}=0$ and $z_{o} / a=10^{-15}$. The values of angular momentum and energy are $\ell / \sqrt{a G m}=0.3, a E / G m=-0.73$, giving $\Delta E=3.9 \times 10^{-3}$. The ratio between the vertical and radial average periods is $T_{z} / T_{R} \approx 0.05$. It was calculated from the peaks in the time series of $z(t)$ and $R(t)$. The predicted envelopes from Eq. (11) are shown by black lines. In our choice, the predicted and numerically calculated envelopes have the same value at the upper point of the zero-velocity curve. The prediction agrees with the envelopes of the numerically integrated orbits. Top, right: Poincaré section in the surface of constant energy and angular momentum given by the orbit's parameters, with $R / a=0.62$. The consequents of the orbits are calculated for both $P_{R}>0$ and $P_{R}<0$ and are given by black dots, whereas the prediction from the average value of $\tilde{I}_{3, \text { mean }}=1.835 \times 10^{-3}$ is given by the solid black line. We use the dimensionless quantities $\tilde{I}_{3}=(a M)^{-1 / 3} I_{3}$ and $\tilde{t}=\left(G M / a^{3}\right)^{1 / 2} t$. We see that Eq. (12) is a good description for the approximate third integral in low-amplitude orbits. Bottom, left: Time series of $I_{3}(t)$, given by Eq. (12). It shows small spread along time evolution. Bottom, right: Histogram for $\tilde{I}_{3}(t)$.

\subsection{Orbits in Kuzmin disk}

The potential-density pair for the Kuzmin disk, $\left(\Phi_{k}, \Sigma_{k}\right)$, is given by (Binney and Tremaine 2008)

$$
\Phi_{k}=-\frac{G m}{\sqrt{R^{2}+(|z|+a)^{2}}}, \quad \Sigma_{k}=\frac{m}{2 \pi a^{2}}\left(1+\frac{R^{2}}{a^{2}}\right)^{-3 / 2},
$$

where $m$ is the total mass of the disk and $a$ is a positive parameter representing the radial length scale. Figure 1 top shows the potential level contours for $\Phi_{k}$ and the 

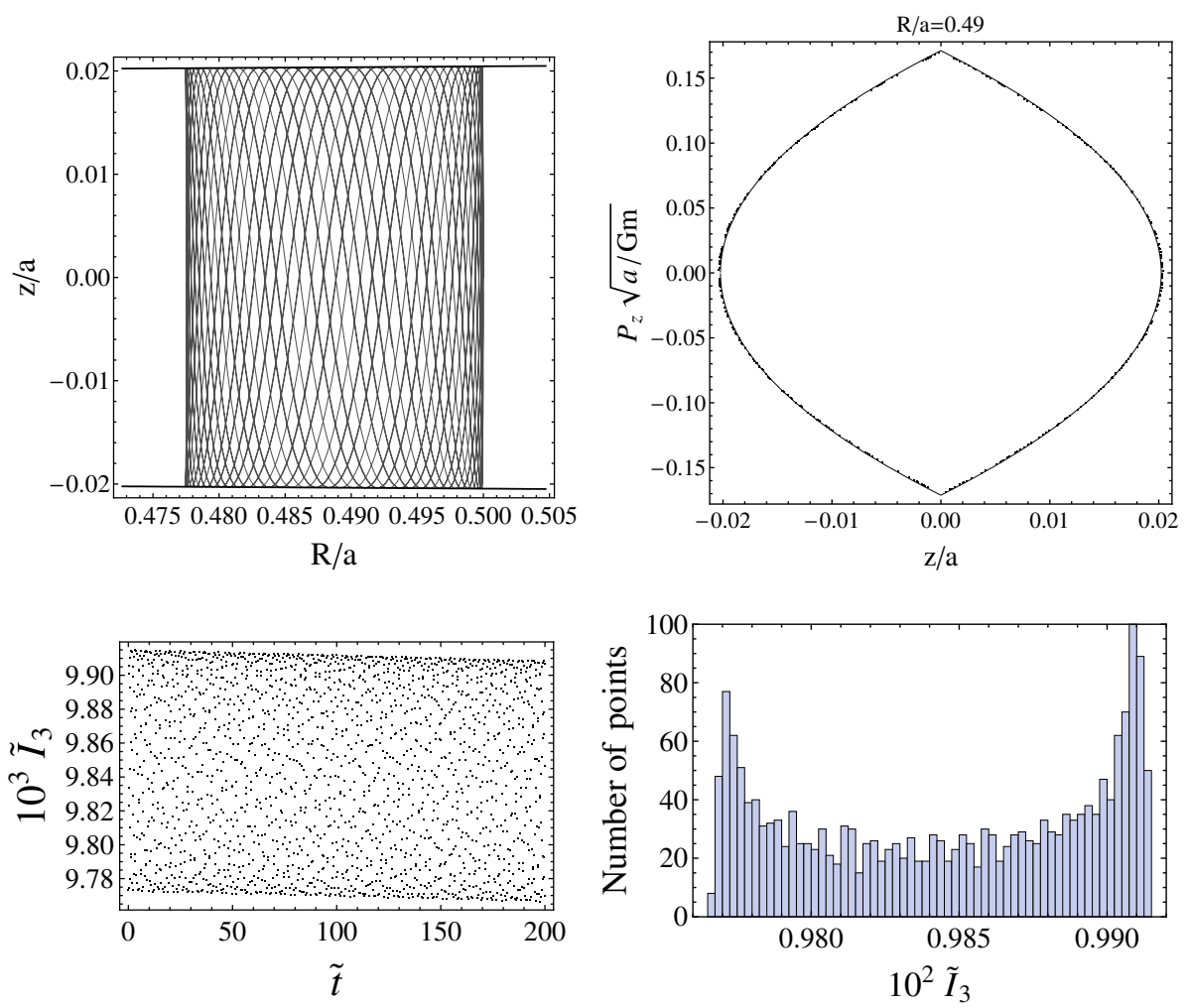

Fig. 3 Orbit in Kuzmin's potential with $R_{o} / a=0.5, P_{R}=0$ and $z_{o} / a=10^{-9}$. The values of angular momentum and energy are $\ell / \sqrt{a G m}=0.2, a E / G m=-0.8$, giving $\Delta E=1.8 \times$ $10^{-2}$. We have $T_{z} / T_{R} \approx 0.15$. The Figure's style is the same as in Fig. 2. The Poincaré section is calculated for $R / a=0.49$. We see that the prediction of Eq. (12) is still a good approximation for this value of vertical amplitude and $\Delta E$. The spread in $I_{3}(t)$ remains small and the consequents in the Poincaré section lie practically on the curve predicted by $\tilde{I}_{3, \text { mean }}=$ $9.845 \times 10^{-3}$.

$R$ - and $z$-components of the corresponding gravitational field. We obtain, besides accurate predictions for low-amplitude orbits (Figs. 2 and 3), very energetic orbits whose envelopes are well described by (11) (Figs. 4, 5 and 6). These orbits can span a considerable radial range, and their vertical amplitudes may be comparable to the radial length scale of the disk, $Z / a \lesssim 1$. All orbits of Figs. $2-6$ have $T_{z} / T_{R}<1$, calculated via the number of peaks in the time series for $z(t)$ and $R(t)$, where $T_{z}\left(T_{R}\right)$ is the vertical (radial) average period of oscillation, meaning that we are in the region where the adiabatic assumption is valid. The precise reason for this unexpectedly wide range of validity for expressions (11)-(12) may be linked to the fact that the Kuzmin potential gives rise to an integrable Hamiltonian (Hunter 2005). We see next that, when considering a different razor-thin disk model, the range of validity of (11)-(12) decreases abruptly. 

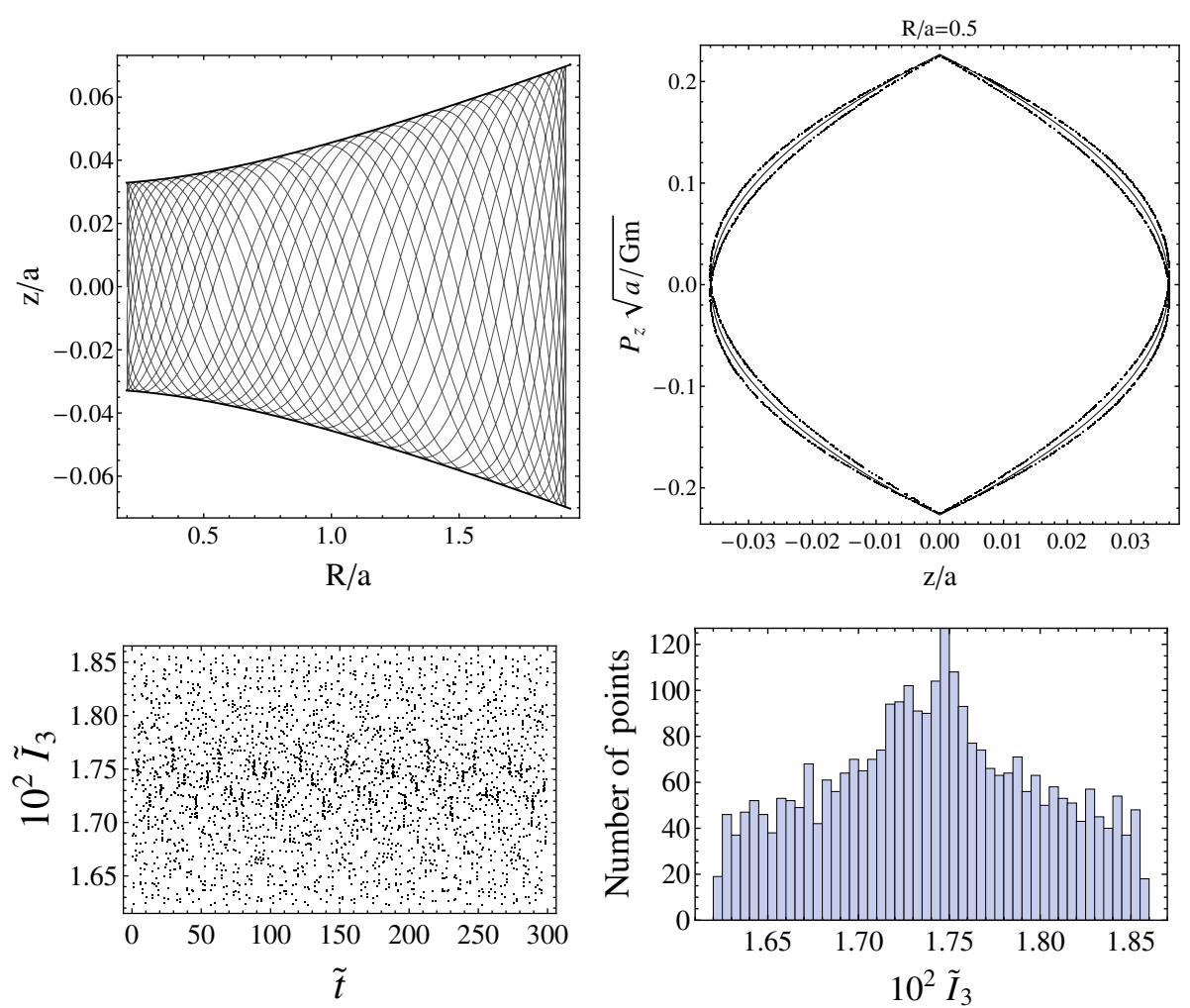

Fig. 4 Orbit in Kuzmin's potential with $R_{o} / a=0.2, P_{R}=0$ and $z_{o} / a=10^{-10}$. The values of angular momentum and energy are $\ell / \sqrt{a G m}=0.2, a E / G m=-0.45$, giving $\Delta E=0.4477$. We have $T_{z} / T_{R}=0.29$. The Figure's style is the same as in Fig. 2 . We see that the spread in the approximate third integral is larger than in Figs. 2 and 3, because the orbits are deviating from quasi-circular motion. The Poincaré section is calculated for $R / a=0.5$ and $\tilde{I}_{3 \text {,mean }}=$ $1.74 \times 10^{-2}$. The consequents of the orbit are different for $P_{R}>0$ and $P_{R}<0$, since the orbit has a larger vertical amplitude (see e.g. Binney and McMillan 2011).

\subsection{Orbits in Generalized Kalnajs disk $m=2$}

As a second example, we choose the second member of the generalized Kalnajs disks (González and Reina 2006), whose surface mass density is a monotonically decreasing function of radius,

$$
\Sigma=\frac{5 M}{2 \pi a^{2}}\left(1-\frac{R^{2}}{a^{2}}\right)^{3 / 2},
$$

where $a$ is the radius of the disk and $M$ is its total mass. The corresponding gravitational potential can be cast in oblate spheroidal coordinates, $\xi=$ $a^{-1} \operatorname{Re}\left[\sqrt{R^{2}+(z-\mathrm{i} a)^{2}}\right], \eta=-a^{-1} \operatorname{Im}\left[\sqrt{R^{2}+(z-\mathrm{i} a)^{2}}\right]$, through the relation

$$
\begin{aligned}
\Phi_{K 2}=-\frac{G M}{a}\left[\cot ^{-1} \xi\right. & +A\left(3 \eta^{2}-1\right) \\
& \left.+B\left(35 \eta^{4}-30 \eta^{2}+3\right)\right],
\end{aligned}
$$



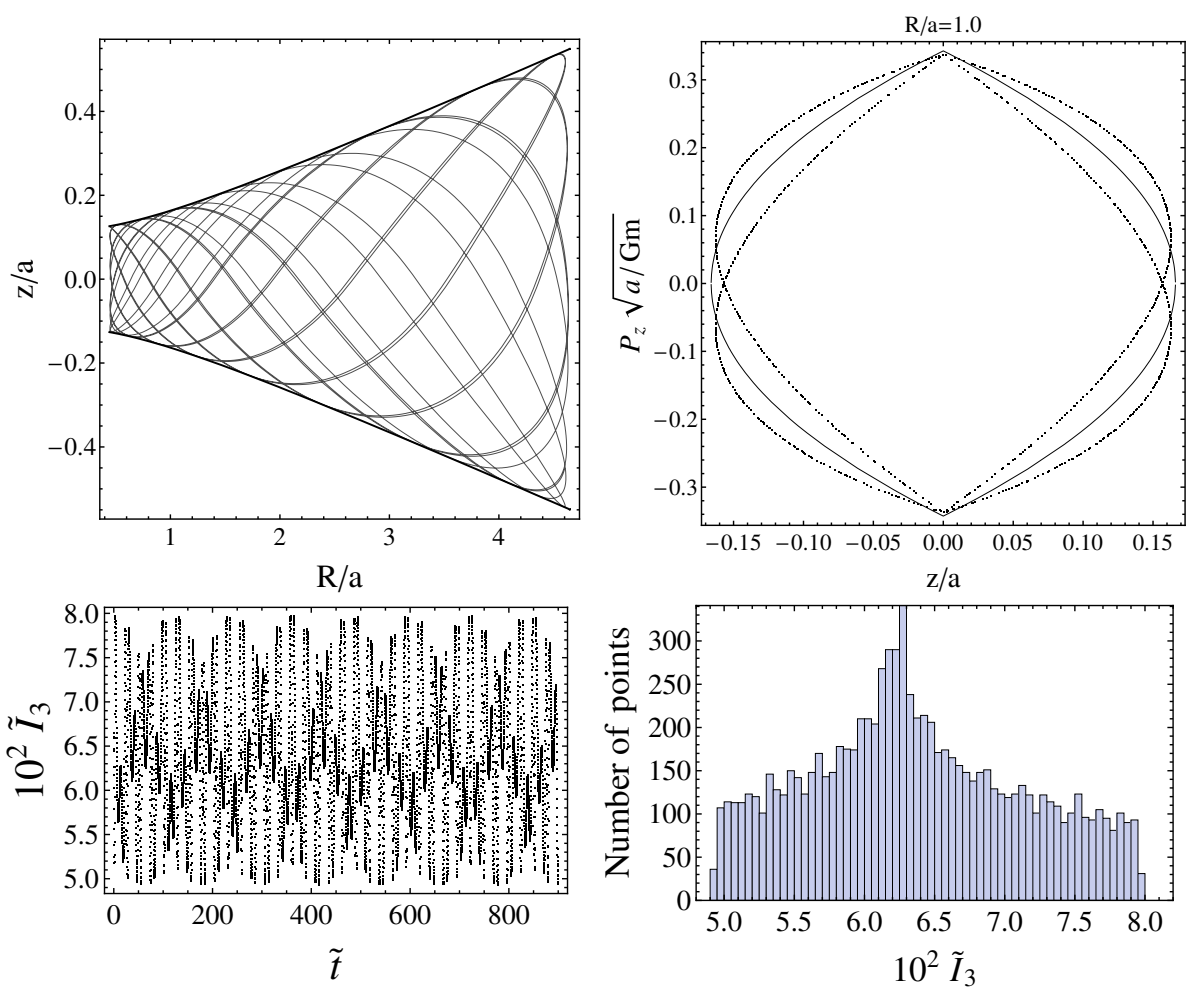

Fig. 5 Orbit in Kuzmin's potential with $R_{o} / a=0.45, P_{R}=0$ and $z_{o} / a=10^{-10}$. The values of angular momentum and energy are $\ell / \sqrt{a G m}=0.5, a E / G m=-0.2$, giving $\Delta E=0.6606$. We have $T_{z} / T_{R} \approx 0.54$. The Figure's style is the same as in Fig. 2. The Poincaré section is calculated for $R / a=1.0$. We see that the spread in the approximate third integral is larger than in Fig. 4. The difference between the consequents of the orbit in the Poincaré section and the prediction from $\tilde{I}_{3 \text {, mean }}=6.36 \times 10^{-2}$ also increases comparing to Fig. 4 . This is due, among other factors, to the deviations from quasi-circular motion and the fact that $T_{z} / T_{R}$ approaches 1 .

with

$$
\begin{aligned}
& A=\frac{5}{14}\left[\left(3 \xi^{2}+1\right) \cot ^{-1} \xi-3 \xi\right] \\
& B=\frac{3}{448}\left[\left(35 \xi^{4}+30 \xi^{2}+3\right) \cot ^{-1} \xi-35 \xi^{3}-\frac{55}{3} \xi\right] .
\end{aligned}
$$

The potential level contours and force field are shown in Fig. 1 bottom. This potential leads to a rotation curve which has a maximum inside the disk, in contrast with the first member of the family (the well-known Kalnajs disk), which describes a configuration rotating as a rigid body.

It is known that motion in this potential presents chaotic behavior (RamosCaro et al. 2008). Numerical experiments show that the range of applicability of (11)-(12) in this potential is much smaller than in the Kuzmin disk. The predictions for $Z(R)$ and $I_{3}$ remain valid for $\Delta E \ll 1$, corresponding to orbits with very small radial and vertical amplitudes (Fig. 7). For low angular momentum (such 

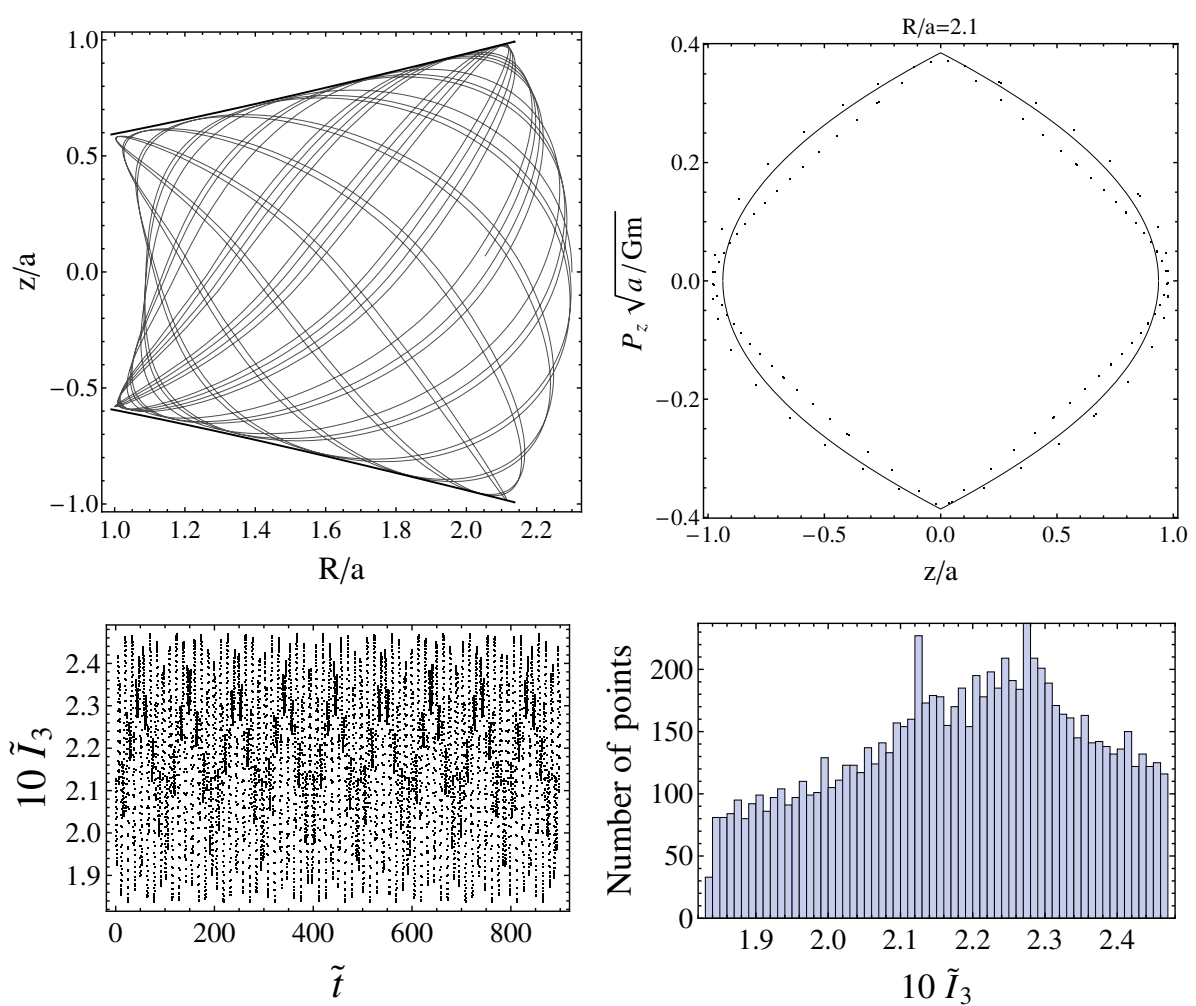

Fig. 6 Orbit in Kuzmin's potential with $R_{o} / a=2.3, P_{R}=0$ and $z_{o} / a=10^{-10}$. The values of angular momentum and energy are $\ell / \sqrt{a G m}=0.7, a E / G m=-0.29$, giving $\Delta E=0.384$. We have $T_{z} / T_{R} \approx 0.91$. The Figure's style is the same as in Fig. 2. The Poincaré section is calculated for $R / a=2.1$ and $\tilde{I}_{3, \text { mean }}=0.2181$.

that the orbit is confined to the inner regions of the disk), orbits with small radial span may have higher vertical amplitudes and still satisfy Eqs. (11)-(12) (see Fig. 8). However, once the radial span of the orbit is increased, equation (11) is not sufficient anymore to describe the orbits' envelopes (see Fig. 9, where the orbit has the same value of $\Delta E$ as in Fig. 8). As the angular momentum increases (still keeping the orbits in the disk region $R<a$ ), the range of validity of the prediction from adiabatic invariance decreases considerably, being valid only for orbits with small enough vertical amplitudes. All orbits of Figs. $7-9$ have $T_{z} / T_{R}<1$.

\subsection{Orbits in Kuzmin disk + Plummer halo}

Now we consider the case corresponding to the superposition of a Kuzmin disk (eq. (14)) and a Plummer halo, whose potential-density pair is given by (Binney and Tremaine 2008)

$$
\Phi_{p}=-\frac{G M}{\sqrt{r^{2}+b^{2}}}, \quad \rho_{p}=\frac{3 M}{4 \pi b^{3}}\left(1+\frac{r^{2}}{b^{2}}\right)^{-5 / 2},
$$



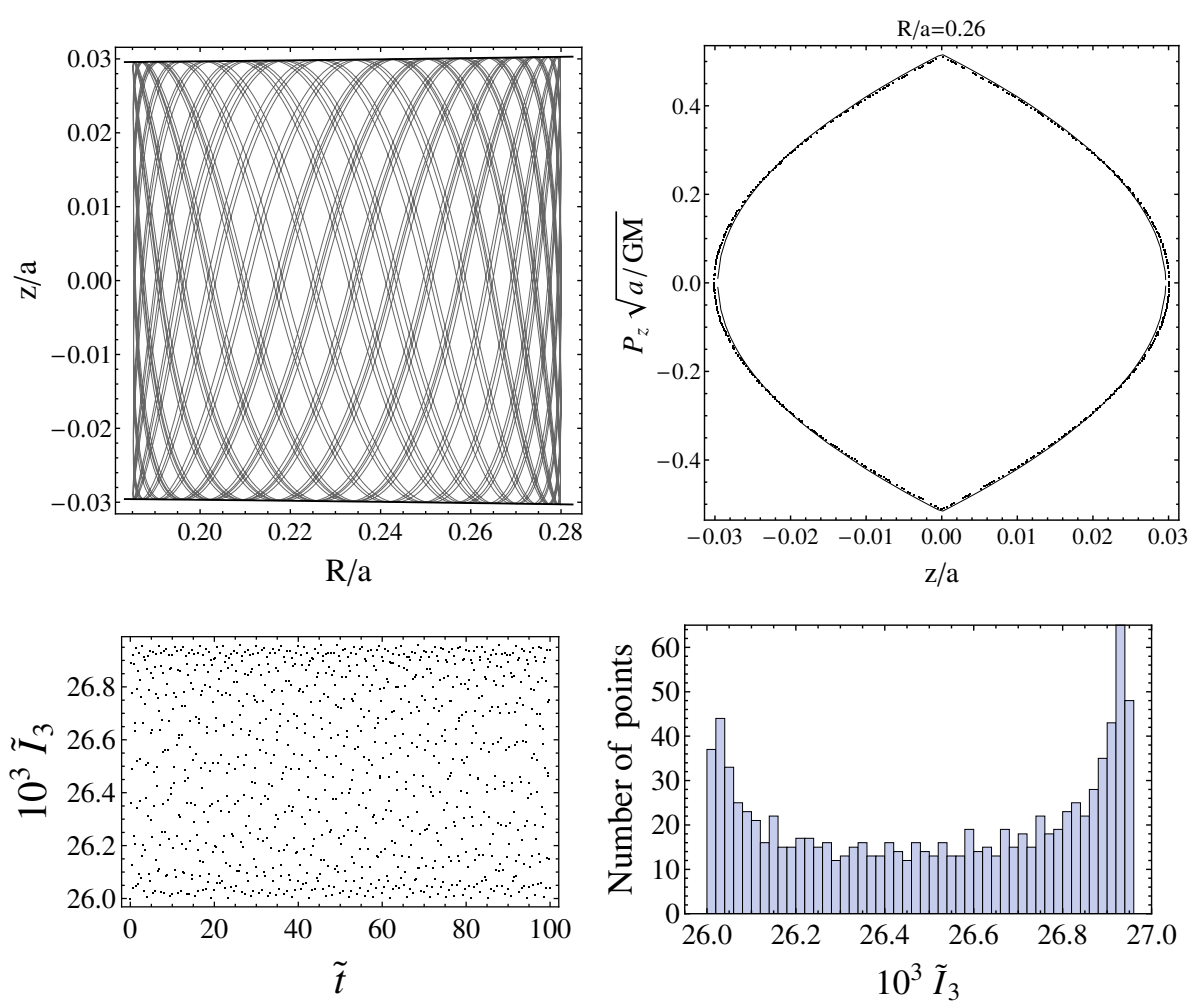

Fig. 7 Orbit in Kalnajs $(m=2)$ potential with $R_{o} / a=0.28, P_{R}=0$ and $z_{o} / a=10^{-8}$. The values of angular momentum and energy are $\ell / \sqrt{a G M}=0.12, a E / G M=-2.5$, giving $\Delta E=0.059$. We have $T_{z} / T_{R} \approx 0.33$. The Figure's style is the same as in Fig. 2 . The Poincaré section is calculated for $R / a=0.26$ and $\tilde{I}_{3, \text { mean }}=0.0269$.

where $r^{2}=R^{2}+z^{2}$. We find a variety of orbits (e.g. Fig. 10), with sufficiently low energy, whose envelopes are well described by relation (11). This was obtained for different combinations of the parameters $M / m$ and $b / a$, even for the case in which the mass of the Plummer sphere is not negligible, as in Fig. 11, where $M / m=0.5$ (lower ratios $M / m$ give a wider region of validity for equation (11)). However, as the halo mass grows, the range of validity of the prediction described above decreases (Fig. 11): deviations from (11) and (12) begin to appear for lower relative energies $\Delta E$, especially in regions where the density of the sphere is higher, $R \lesssim b$. The spherical contribution has a negative impact on the range of validity of the predictions for $Z(R)$ and $I_{3}$, what may be explained by the fact that (11)-(12) take into account only the razor-thin disk contribution.

\section{Conclusions}

We provide an approach to address the problem of obtaining simple analytical expressions for the third integral of motion, associated with a great variety of disk-crossing orbits in galactic models incorporating a razor-thin disk. The ap- 

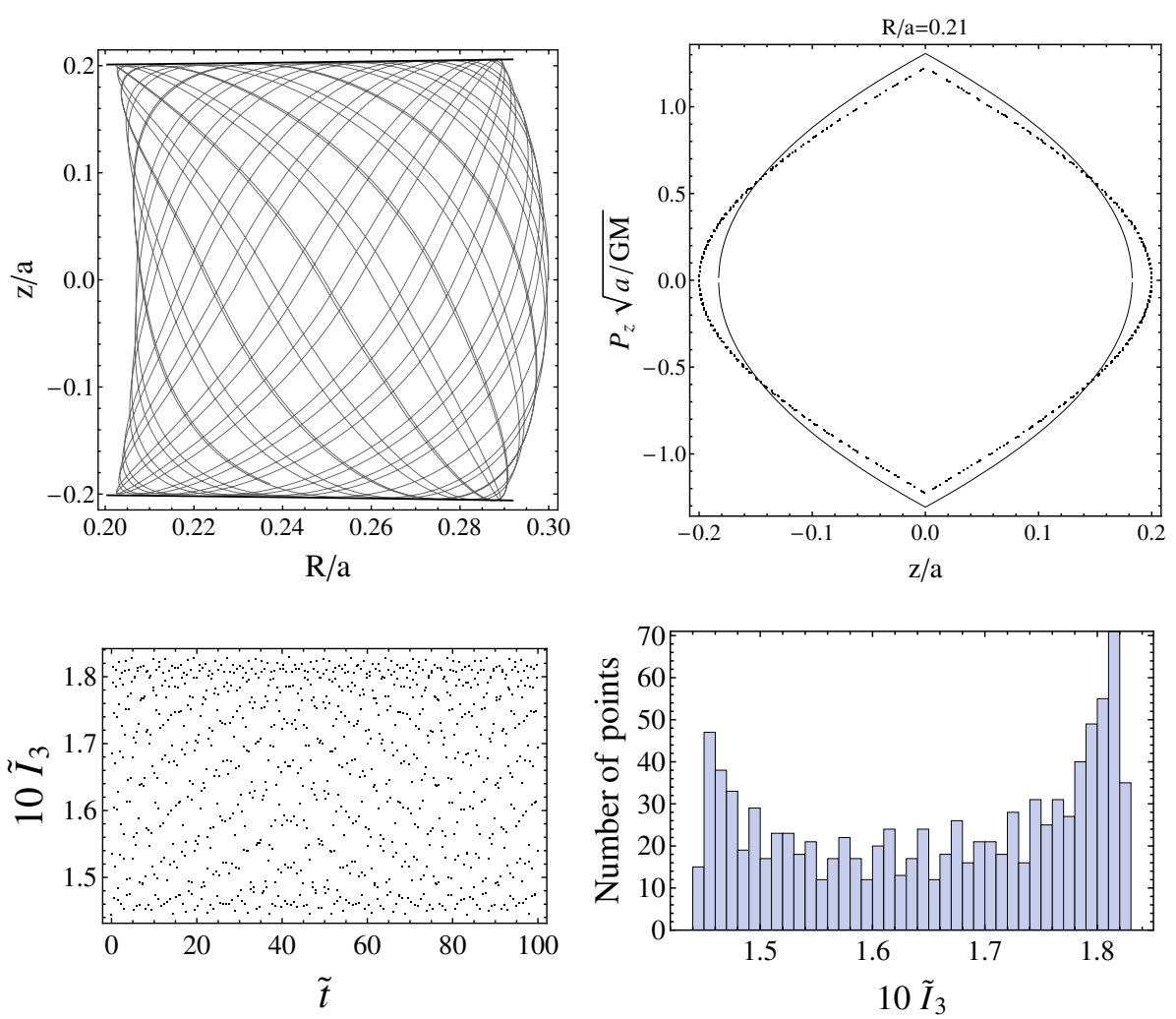

Fig. 8 Orbit in Kalnajs $(m=2)$ potential with $R_{o} / a=0.3, P_{R}=0$ and $z_{o} / a=10^{-8}$. The values of angular momentum and energy are $\ell / \sqrt{a G M}=0.12, a E / G M=-1.9$, giving $\Delta E=0.285$. We have $T_{z} / T_{R} \approx 0.91$. The Figure's style is the same as in Fig. 2 . The Poincaré section is calculated for $R / a=0.21$ and $\tilde{I}_{3, \text { mean }}=0.1656$.

proximated third integral of motion is given by Eq. (12) with the orbits' envelopes given by (11), and, in general, this is valid for orbits with small vertical amplitude. However, numerical experiments also reveal that the quantity $I_{3}$ (see Eq. (12)) is approximately conserved for a variety of orbits with larger values of $Z$ (i.e. of the order of the system's characteristic radius). The same behavior was observed in models where the stellar distribution is represented by a thickened disk (see Vieira and Ramos-Caro 2014). It is worth pointing out that Eq. (12) is also valid in the presence of a 3D distribution surrounding the razor-thin disk, such as a thick disk or a halo. However, as the mass of this 3D-component grows, the predictions of (12) tend to be less accurate, since it only takes into account the potential of the razor-thin component.

A fundamental step in obtaining formula (12) was the study on the stability of equatorial circular orbits under small vertical perturbations, in models incorporating an equatorial discontinuity of the gravitational field. We find that, in every galactic razor-thin disk model, all the equatorial circular orbits inside the matter distribution are vertically stable. It leads us to point out that all circular orbits of the thin-disk models presented in González and Reina (2006); Letelier 

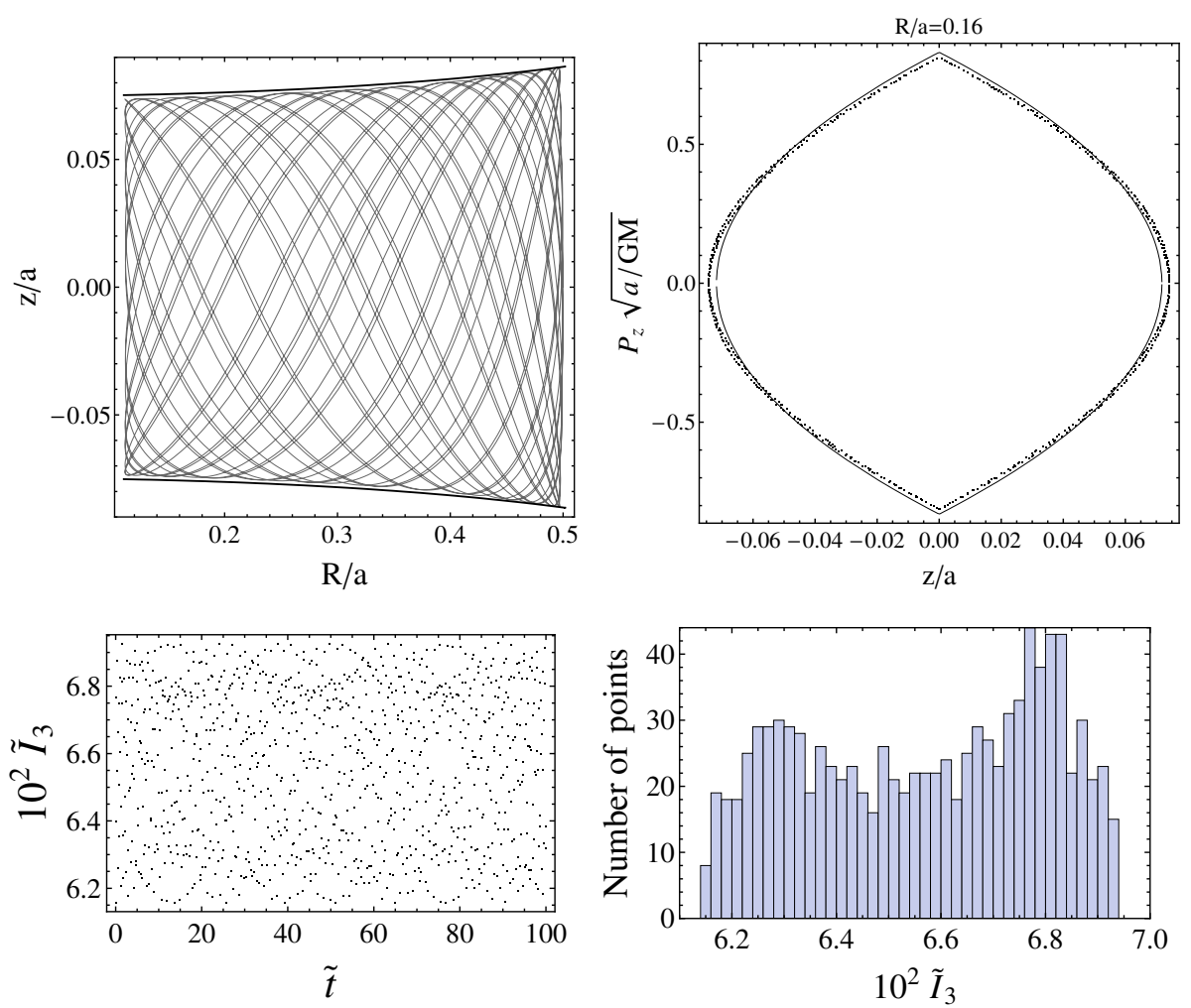

Fig. 9 Orbit in Kalnajs $(m=2)$ potential with $R_{o} / a=0.5, P_{R}=0$ and $z_{o} / a=10^{-8}$. The values of angular momentum and energy are $\ell / \sqrt{a G M}=0.12, a E / G M=-2$, giving $\Delta E=0.285$. We have $T_{z} / T_{R} \approx 0.54$. The Figure's style is the same as in Fig. 2 . The Poincaré section is calculated for $R / a=0.16$ and $\tilde{I}_{3, \text { mean }}=6.656 \times 10^{-2}$.

(2007); Vogt and Letelier (2009); González et al. (2010); Ramos-Caro et al. (2008, 2011); Pedraza et al. (2008) are stable, contrary to the statements made in such references.

We obtained the formula described above via a first-order approximation in $|z|$ to the vertical dependence of the effective potential, in the vicinity of the equatorial plane. A better analytical description of off-equatorial orbits in systems containing razor-thin disks would be obtained if we extended this approximate third invariant in a power series of $x=R-R_{o}$ and $|z|$. This procedure would be the razorthin version of the smooth-case Contopoulos' third integral (Contopoulos 1960, 1963). However, such a procedure is far from being a direct extension of the firstorder case we derived here and is beyond the scope of this work. The subtleties of the $z=0$ discontinuity do not allow us to determine the expansions of $H$ and $I_{3}$ in a clear, straightforward manner, since the terms involving $\sum_{m, k} x^{m}|z|^{k}$ (k odd) and $\sum_{j} x^{N-2 j}|z|^{2 j}$ (with $m+k=N-1$ ) would be comparable. The corresponding arbitrary-order expansion is under investigation, and will be the subject of a forthcoming paper. 

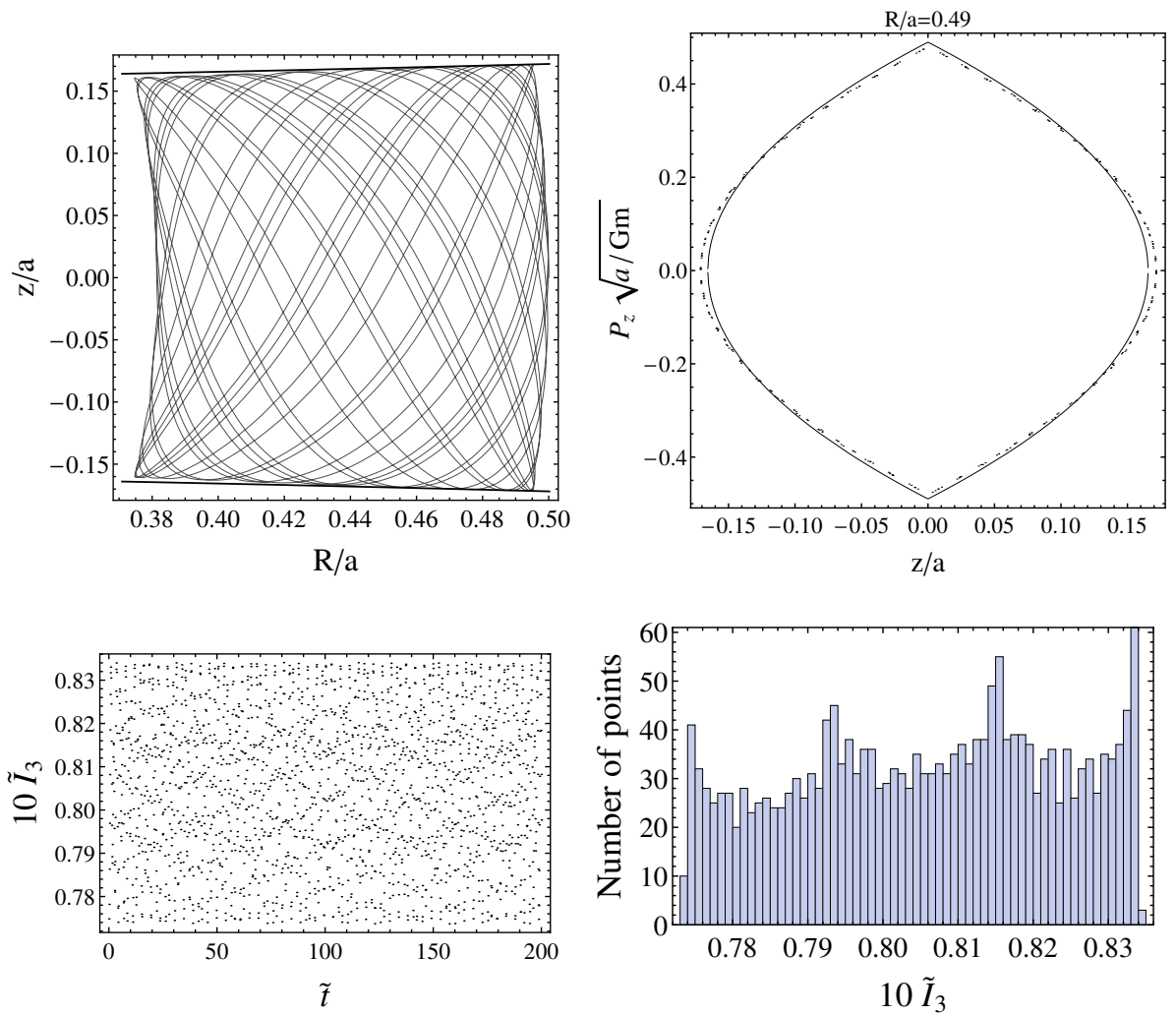

Fig. 10 Orbit with initial conditions $R_{o} / a=0.5, P_{R}=0$ and $z_{o} / a=10^{-9}$, in a potential due to the superposition of a Kuzmin disk and a Plummer halo such that $M / m=0.05$ and $b / a=1.5 \times 10^{-2}$. The values of angular momentum and energy, as well as the initial conditions, are the same as in Fig. 3: $\ell / \sqrt{a G m}=0.2, a E / G m=-0.8$, giving in this case $\Delta E=1.38 \times 10^{-1}$ and $T_{z} / T_{R} \approx 0.65$. The initial conditions and orbit's parameters are the same as the parameters of Fig. 3; however, the presence of the halo increases the orbit's vertical amplitude by one order of magnitude. The Poincaré section is also calculated for $R / a=0.49$. We see that the prediction of Eq. (12) is a good approximation for this value of vertical amplitude and $\Delta E$. The spread in $I_{3}(t)$ grows, in relation with fig. 3 , and the consequents in the Poincaré section deviate a little from the curve predicted by $\tilde{I}_{3, \text { mean }}=8.057 \times 10^{-2}$.

The existence of a third integral of motion has a central role in the formulation of self-consistent galactic models, in which the distribution function (DF), solution to the collisionless Boltzmann equation, is known. As a consequence of Jeans theorem, any equilibrium DF can be written as a function of the integrals of motion (Binney and Tremaine 2008), i.e. an expression of the form $f\left(E, \ell, I_{3}\right)$, where $I_{3}$ is the non-classical third integral. Therefore, one interesting consequence of Eq. (12) (which works very well for orbits with small vertical amplitude) is that a DF of the form $f\left(E, \ell, I_{3}\right)$ could provide a satisfactory statistical representation for flattened 3D self-gravitating configurations (Vieira and Ramos-Caro 2014). Another interesting consequence of relation (12) is the possibility of having at hand an alternative (or complementary) method to measure the mass distribution of the galactic disk, in this case, from observations determining the vertical amplitudes 

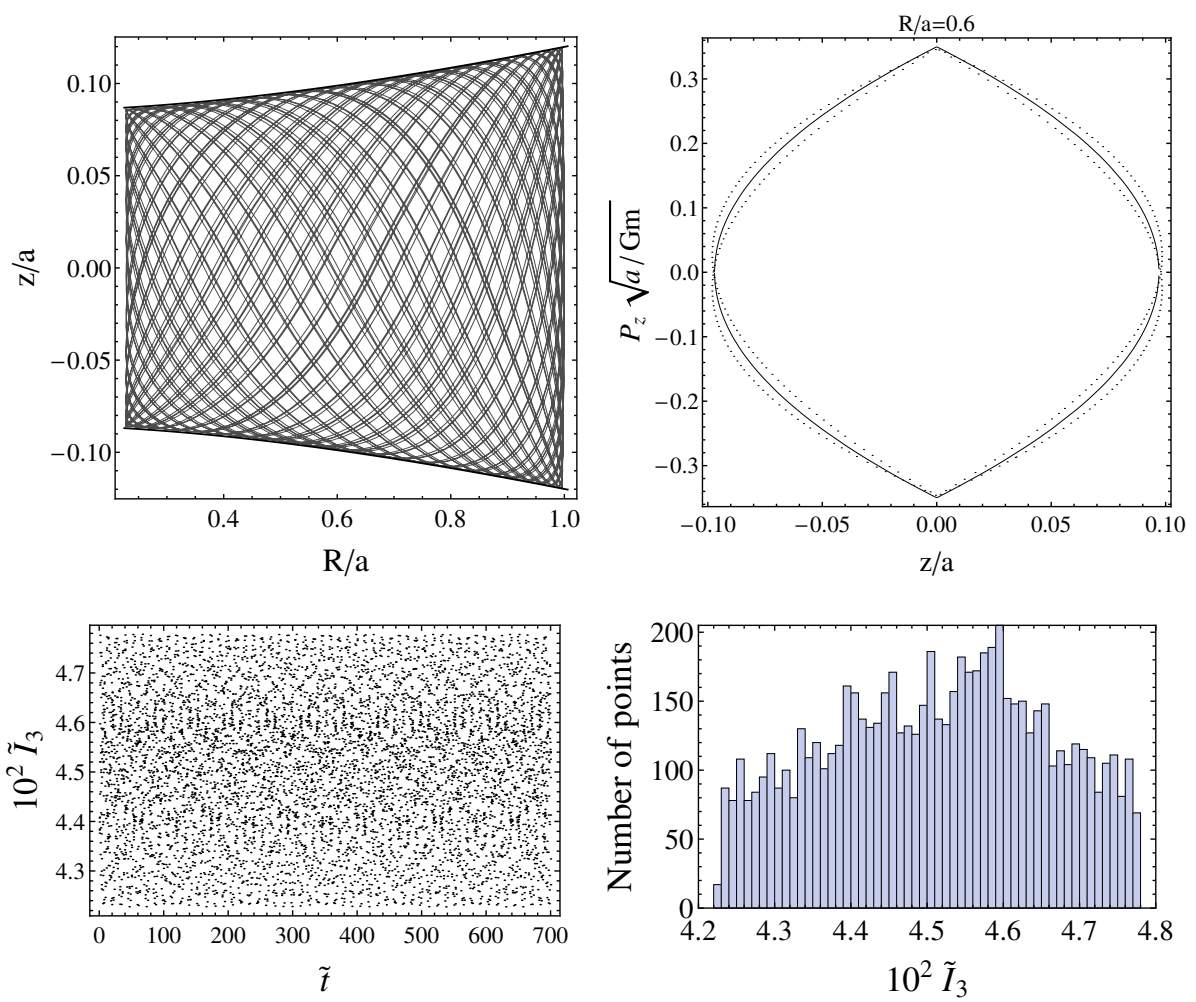

Fig. 11 Orbit in the Kuzmin + Plummer potential, with initial conditions $R_{o} / a=1.0$, $P_{R}=0, z_{o} / a=10^{-9}, \ell / \sqrt{a G m}=0.2, a E / G m=-1.0$, and system's parameters $M / m=0.5$ and $b / a=1$, giving $\Delta E=2.125 \times 10^{-1}$ and $T_{z} / T_{R} \approx 0.61$. The Poincaré section is also calculated for $R / a=0.6$. We see that the prediction of Eq. (12) remains a good approximation for this value of vertical amplitude and $\Delta E$, even when the ratio $M / m$ is one order of magnitude greater than in Fig. 10. The spread in $I_{3}(t)$ grows, in relation with Fig. 10, however the consequents in the Poincaré section remain close to the curve predicted by $\tilde{I}_{3, \text { mean }}=4.512 \times$ $10^{-2}$.

of disk-crossing orbits. This procedure may be applicable, for instance, to orbits belonging to the thick-disk component of a given galaxy mass model, whose thindisk component is modeled as having zero thickness. It is also applicable to orbits in other three-dimensional distributions surrounding a razor-thin disk, e.g. the Kuzmin + Plummer system studied in sec. 5.3.

\section{Acknowledgements}

The work of RSSV is supported by the "São Paulo Research Foundation" (FAPESP), grants 2010/00487-9 and 2015/10577-9. RSSV thanks Alberto Saa for fruitful discussions and Sylvio Ferraz-Mello for helpful remarks on the accepted version of the manuscript. The authors thank the anonymous referees for insightful comments which helped us improve the final version of the manuscript. The authors also acknowledge the support from FAPESP grant 2013/09357-9. 


\section{References}

Bienaymé, O., Robin, A.C., Famaey, B. Quasi integral of motion for axisymmetric potentials. Astron. Astrophys.581, A123, 2015. doi:10.1051/0004$6361 / 201526516$

Bienaymé, O., Traven, G. Approximate integrals of motion. Astron. Astrophys.549, A89, 2013. doi:10.1051/0004-6361/201220008

Binney, J. Distribution functions for the Milky Way. Mon. Not. R. Astron. Soc.401, 2318-2330, 2010. doi:10.1111/j.1365-2966.2009.15845.x

Binney, J. More dynamical models of our Galaxy. Mon. Not. R. Astron. Soc.426, 1328-1337, 2012. doi:10.1111/j.1365-2966.2012.21692.x

Binney, J., McMillan, P. Models of our Galaxy - II. Mon. Not. R. Astron. Soc.413, 1889-1898, 2011. doi:10.1111/j.1365-2966.2011.18268.x

Binney, J., Sanders, J.L. Dynamical models and Galaxy surveys. In S. Feltzing, G. Zhao, N.A. Walton, P. Whitelock (eds.) IAU Symposium, volume 298 of IAU Symposium, pages 117-129. 2014. doi:10.1017/S1743921313006297

Binney, J., Tremaine, S. Galactic Dynamics. Princeton Univ. Press, Princeton, NJ, second edition, 2008

Contopoulos, G. A third Integral of Motion in a Galaxy. Z. Astrophys.49, 273, 1960

Contopoulos, G. On the existence of a third integral of motion. Astron. J.68, 1, 1963. doi:10.1086/108903

Contopoulos, G. The development of nonlinear dynamics in astronomy. Foundations of Physics 31, 89-114, 2001. doi:10.1023/A:1004155905361

Contopoulos, G. Order and chaos in dynamical astronomy. Springer-Verlag, 2002

de Zeeuw, P.T. Dynamical models for axisymmetric and triaxial galaxies. In P.T. de Zeeuw (ed.) Structure and Dynamics of Elliptical Galaxies, volume 127 of IAU Symposium, pages 271-289. 1987

Freeman, K.C. On the Disks of Spiral and so Galaxies. Astrophys. J.160, 811, 1970. doi:10.1086/150474

González, G.A., Plata-Plata, S.M., Ramos-Caro, J. Finite thin disc models of four galaxies in the UrsaMajor cluster: NGC3877, NGC3917, NGC3949 and NGC4010. Mon. Not. R. Astron. Soc.404, 468-474, 2010. doi:10.1111/j.13652966.2010.16303.x

González, G.A., Reina, J.I. An infinite family of generalized Kalnajs discs. Mon. Not. R. Astron. Soc.371, 1873-1876, 2006. doi:10.1111/j.1365-2966.2006.10819.x

Hietarinta, J. Direct methods for the search of the second invariant. Phys. Rep.147, 87-154, 1987. doi:10.1016/0370-1573(87)90089-5

Hunter, C. The structure and stability of self-gravitating disks. Mon. Not. R. Astron. Soc.126, 299, 1963. doi:10.1093/mnras/126.4.299

Hunter, C. Disk-Crossing Orbits. In G. Contopoulos, N. Voglis (eds.) Galaxies and Chaos, volume 626 of Lecture Notes in Physics, Berlin Springer Verlag, pages 137-153. 2003. doi:10.1007/978-3-540-45040-5_11

Hunter, C. Chaos in Orbits Due to Disk Crossings. Annals of the New York Academy of Sciences 1045, 120, 2005. doi:10.1196/annals.1350.011

Iorio, L. Orbital Perturbations Due to Massive Rings. Earth Moon and Planets 108, 189-217, 2012. doi:10.1007/s11038-012-9391-1

Kent, S.M. Dark matter in spiral galaxies. I - Galaxies with optical rotation curves. Astron. J.91, 1301-1327, 1986. doi:10.1086/114106 
Kent, S.M. Dark matter in spiral galaxies. II - Galaxies with H I rotation curves. Astron. J.93, 816-832, 1987. doi:10.1086/114366

Landau, L.D., Lifshitz, E.M. Fluid Mechanics, volume VI of Course of Theoretical Physics. Elsevier, Oxford, UK, second edition, 2007

Lemos, J.P.S., Letelier, P.S. Exact general relativistic thin disks around black holes. Phys. Rev. D49, 5135-5143, 1994. doi:10.1103/PhysRevD.49.5135

Letelier, P.S. Stability of circular orbits of particles moving around black holes surrounded by axially symmetric structures. Phys. Rev. D68, 104002, 2003. doi:10.1103/PhysRevD.68.104002

Letelier, P.S. Simple potential-density pairs for flat rings. Mon. Not. R. Astron. Soc.381, 1031-1034, 2007. doi:10.1111/j.1365-2966.2007.12128.x

Lora-Clavijo, F.D., Ospina-Henao, P.A., Pedraza, J.F. Charged annular disks and Reissner-Nordström type black holes from extremal dust. Phys. Rev. D82, 084005, 2010. doi:10.1103/PhysRevD.82.084005

Morgan, T., Morgan, L. The Gravitational Field of a Disk. Physical Review 183, 1097-1101, 1969. doi:10.1103/PhysRev.183.1097

Pedraza, J.F., Ramos-Caro, J., González, G.A. An infinite family of self-consistent models for axisymmetric flat galaxies. Mon. Not. R. Astron. Soc.390, 1587-1597, 2008. doi:10.1111/j.1365-2966.2008.13846.x

Ramos-Caro, J., López-Suspes, F., González, G.A. Chaotic and regular motion around generalized Kalnajs discs. Mon. Not. R. Astron. Soc.386, 440-446, 2008. doi:10.1111/j.1365-2966.2008.13047.x

Ramos-Caro, J., Pedraza, J.F., Letelier, P.S. Motion around a monopole + ring system - I. Stability of equatorial circular orbits versus regularity of three-dimensional motion. Mon. Not. R. Astron. Soc.414, 3105-3116, 2011. doi:10.1111/j.1365-2966.2011.18618.x

Saa, A., Venegeroles, R. Chaos around the superposition of a black-hole and a thin disk. Physics Letters A 259, 201-206, 1999. doi:10.1016/S0375-9601(99)00447-8

Semerák, O., Suková, P. Free motion around black holes with discs or rings: between integrability and chaos - I. Mon. Not. R. Astron. Soc.404, 545-574, 2010. doi:10.1111/j.1365-2966.2009.16003.x

Sofue, Y., Honma, M., Omodaka, T. Unified Rotation Curve of the Galaxy - Decomposition into de Vaucouleurs Bulge, Disk, Dark Halo, and the 9-kpc Rotation Dip -. Publ. Astron. Soc. Jpn.61, 227-, 2009. doi:10.1093/pasj/61.2.227

Varvoglis, H. Non ergodic particle motion in a c0 potential. Journal de Physique 46, 495-502, 1985

Vieira, R.S.S., Ramos-Caro, J. A Simple Formula for the Third Integral of Motion of Disk-crossing Stars in the Galaxy. Astrophys. J.786, 27, 2014. doi:10.1088/0004-637X/786/1/27

Vieira, R.S.S., Ramos-Caro, J. On the stability of circular orbits in galactic dynamics: Newtonian thin disks. In K. Rosquist, R.T. Jantzen, R. Ruffini (eds.) The Thirteenth Marcel Grossmann Meeting, pages 2346-2348. World Scientific, 2015. doi:10.1142/9789814623995_0438

Vogt, D., Letelier, P.S. Analytical potential-density pairs for flat rings and toroidal structures. Mon. Not. R. Astron. Soc.396, 1487-1498, 2009. doi:10.1111/j.13652966.2009.14803.x 